\title{
Long-Term Outcomes of Open Surgery and Stent Graft Treatment in Patients Undergoing Repeat Thoracic Aortic Aneurysm Repair from Previous Anastomosis Site
}

\author{
Daijiro Hori, MD, PhD, Koichi Yuri, MD, PhD, Sho Kusadokoro, MD, \\ Hiroyasu Katayama, MD, Naoyuki Kimura, MD, PhD, and Atsushi Yamaguchi, MD, PhD
}

Objective: The purpose of this study was to compare the long-term outcomes of open surgery and thoracic endovascular aortic repair (TEVAR) in patients undergoing repeat thoracic aortic repair from previous anastomosis site.

Methods: From January 2009 to December 2017, 68 patients needed repeat aortic surgery from previous anastomosis site. Twenty-three patients had dissected distal aorta and 45 patients had non-dissected distal aorta. Early and long-term outcomes of open surgery and TEVAR were compared in both groups.

Results: There were no significant differences in patient background between the two treatments in both groups. Open surgery was associated with longer intensive care unit stay, but there was no significant difference in in-hospital mortality in both groups. In patients with dissected distal aorta, there was no significant difference in long-term mortality $(p=0.73)$. However, TEVAR was associated with higher risk of reintervention $(p=0.038)$. In non-dissected distal aorta patients, acute kidney injury $(p=0.002)$ and prolonged ventilation $(p=0.032)$ were more often observed in open surgery. However, there were no significant differences in long-term mortality $(p=0.23)$ and freedom from reintervention $(p=0.13)$.

Conclusions: Long-term outcomes were similar between open surgery and TEVAR in both groups. However, TEVAR in patients with dissected distal aorta was associated with

Department of Cardiovascular Surgery, Saitama Medical Center, Jichi Medical University, Saitama, Saitama, Japan

Received: April 25, 2019; Accepted: July 13, 2019

Corresponding author: Daijiro Hori, MD, PhD. Department of Cardiovascular Surgery, Saitama Medical Center, Jichi Medical University, 1-847 Amanuma-cho, Omiya-ku, Saitama, Saitama 330-8503, Japan

Tel: +81-48-647-2111, Fax: +81-48-645-0621

E-mail: dhori07@jichi.ac.jp

(cc) BY-NC-SA (02019 The Editorial Committee of Annals of Vascular Diseases. This article is distributed under the terms of the Creative Commons Attribution License, which permits use, distribution, and reproduction in any medium, provided the credit of the original work, a link to the license, and indication of any change are properly given, and the original work is not used for commercial purposes. Remixed or transformed contributions must be distributed under the same license as the original. higher risk, for reintervention.

Keywords: redo TEVAR, thoracic aorta, aneurysm, redo surgery

\section{Introduction}

Enlargement of the remaining aorta or formation of pseudo-aneurysm at the anastomosis site is often observed after repair of thoracic aortic aneurysm. Aortic arch reoperation rate of $5 \%$ has been reported for those patients who underwent surgery on the ascending aorta with or without involvement of the aortic arch. The indication for repeat surgery included residual arch aneurysm in 50\% of patients, aneurysmal formation due to residual aortic dissection in $38 \%$ of patients, and graft infection in $9 \%$ of patients. ${ }^{1)}$ Furthermore, in a retrospective chart review of 11 European aortic centers, aortic events occurred in $12.6 \%$ of the patients who underwent elective total aortic arch replacement. ${ }^{2}$ )

On the other hand, five years freedom from aneurysmrelated death in patients undergoing elective thoracic endovascular repair (TEVAR) for degenerative thoracic aortic aneurysms is reported to be from $82.4 \%$ to $92.7 \%$. The most common complication among these patients was endoleak, occurring at a rate of $1.4 \%$ to $14.8 \% .^{3)}$

Due to introduction of stent graft technology, surgical strategy for aortic aneurysm is provided with many options. Staged hybrid operation, combining open surgical repair and endovascular repair, has been reported in treating acute type A dissection and extended aortic aneurysms. ${ }^{4,5)}$ Use of stent graft for a repeat thoracic aortic surgery could be another option of surgical strategy for aortic aneurysm.

The purpose of this study was to compare the long-term outcomes of open surgical treatment and TEVAR treatment in patients undergoing repeat thoracic aortic repair from the previous anastomosis site. 


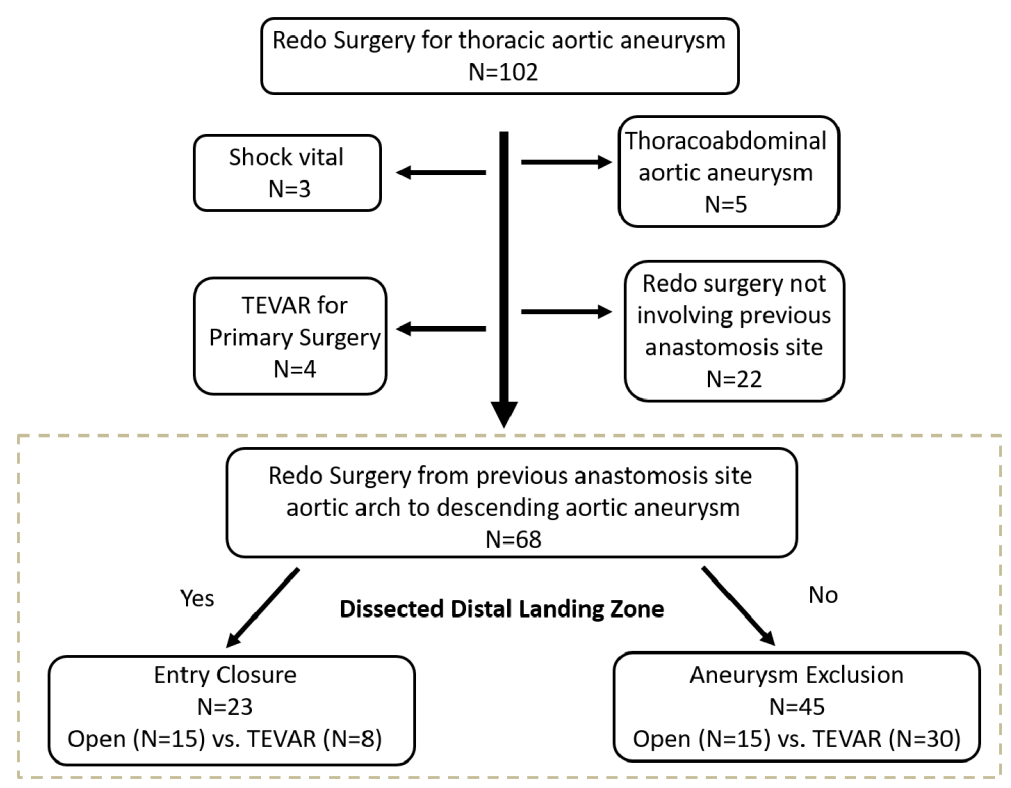

Fig. 1 Flow diagram of the patients included in the study. Sixty-eight patients who underwent repeat surgery from previous anastomosis site were included in the study.

\section{Methods}

From January 2009 to December 2017, there were 102 patients in our institution who underwent repeat aortic surgery of the remaining aortic arch to the descending thoracic aorta. Patients who underwent surgery for thoracoabdominal aortic aneurysm; patients who underwent TEVAR for the initial surgery; patients who were in shock before surgery; and for repeat surgery not involving previous anastomosis site were excluded from the study. The remaining 68 patients who needed treatment from the previous anastomosis site were included in the study (Fig. 1). Of these 68 patients, 23 patients had dissected distal aorta and 45 patients had non-dissected distal aorta (Fig. 2). Outcomes of open surgery and TEVAR were compared in these two groups. The primary goal of TEVAR in patients with dissected distal aorta was closure of the major entry, while exclusion of an aneurysm was achieved in patients with non-dissected distal aorta. The study was approved by the Institutional Review Board of Saitama Medical Center, Jichi Medical University (S18-056).

\section{Statistical analysis}

Normal distribution of the data was assessed using Kolmogorov-Smirnov test. Continuous data that were normally distributed were analyzed using the Student's t-test (mean \pm standard deviation [SD]). Mann-Whitney test (median, interquartile range [IQR]) was used for data that were not normally distributed. For categorical variables, Fisher's exact test was used (n, \%). Early outcomes were compared between the two groups including: hospital

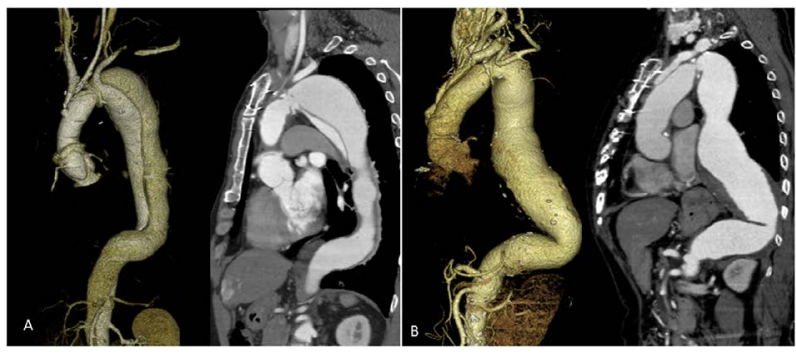

Fig. 2 Representative computed tomography images of patients with dissected distal aorta (A: entry closure group) and non-dissected distal aorta (B: aneurysm exclusion group).

stay; intensive care unit (ICU) stay; in-hospital mortality; sepsis; acute kidney injury; prolonged ventilation; and stroke. Acute kidney injury (AKI) was defined by the KDIGO criteria ${ }^{6}$ and prolonged ventilation was defined as ventilation therapy for more than $48 \mathrm{~h}$ after surgery. For the long-term outcomes, all-cause mortality and freedom rate from reintervention were evaluated using the KaplanMeier curve and log-rank test. $\mathrm{p}<0.05$ was considered significant, and all analysis was performed using Stata (version 13.1, Stata Corp, College Station, TX, USA).

\section{Repeat surgical procedures}

Endovascular treatment was considered in patients with sufficient proximal landing zone of more than $20 \mathrm{~mm}$, with or without debranching of the left axillary artery or left common carotid artery from the right axillary artery. For patients not suitable for endovascular treatment, open surgical repair was considered. For patients who previ- 
Table 1 Patient demographics and early outcomes

\begin{tabular}{|c|c|c|c|c|c|c|}
\hline & \multicolumn{3}{|c|}{ Entry closure } & \multicolumn{3}{|c|}{ Aneurysm exclusion } \\
\hline & Open surgery $n=15$ & TEVAR $n=8$ & $\mathrm{p}$ & Open surgery $n=15$ & TEVAR $n=30$ & $\mathrm{p}$ \\
\hline Age (years) & $62.4 \pm 9.54$ & $64.3 \pm 8.94$ & 0.66 & $67.8 \pm 10.39$ & $69.6 \pm 7.93$ & 0.52 \\
\hline Male (\%) & $9(60.0 \%)$ & $4(50.0 \%)$ & 0.69 & $11(73.3 \%)$ & $24(80.0 \%)$ & 0.71 \\
\hline Angiotensin II receptor blocker (\%) & $10(66.7 \%)$ & $5(62.5 \%)$ & 1 & $7(46.7 \%)$ & $15(50.0 \%)$ & 1 \\
\hline Calcium channel blocker (\%) & $11(73.3 \%)$ & $5(62.5 \%)$ & 0.66 & $11(73.3 \%)$ & $17(56.7 \%)$ & 0.34 \\
\hline Beta blocker (\%) & $14(93.3 \%)$ & $6(75.0 \%)$ & 0.27 & $9(60.0 \%)$ & $18(60.0 \%)$ & 1 \\
\hline HMG-CoA inhibitor (\%) & $2(13.3 \%)$ & $3(37.5 \%)$ & 0.3 & $7(46.7 \%)$ & $16(53.3 \%)$ & 0.76 \\
\hline Anti-platelet (\%) & $3(20.0 \%)$ & $2(25.0 \%)$ & 1 & $4(26.7 \%)$ & $9(30.0 \%)$ & 1 \\
\hline Anti-coagulation (\%) & $4(26.7 \%)$ & $3(37.5 \%)$ & 0.66 & $2(13.3 \%)$ & $8(26.7 \%)$ & 0.46 \\
\hline Creatinine (mg/dl) & $0.95(0.83-1.11)$ & $0.90(0.80-1.94)$ & 0.85 & $1.14(0.82-1.38)$ & $0.92(0.74-1.14)$ & 0.31 \\
\hline Hemoglobin (g/dl) & $12.0 \pm 1.74$ & $11.6 \pm 1.67$ & 0.54 & $12.3 \pm 1.80$ & $11.8 \pm 1.71$ & 0.35 \\
\hline Hyperlipidemia (\%) & $4(26.7 \%)$ & $4(50.0 \%)$ & 0.37 & $7(46.7 \%)$ & $16(53.3 \%)$ & 0.76 \\
\hline Diabetes (\%) & $3(20.0 \%)$ & $0(0.0 \%)$ & 0.53 & $3(20.0 \%)$ & $5(16.7 \%)$ & 1 \\
\hline Hypertension (\%) & $15(100.0 \%)$ & $7(87.5 \%)$ & 0.35 & $15(100.0 \%)$ & $25(83.3 \%)$ & 0.15 \\
\hline Cerebral vascular disease (\%) & $3(20.0 \%)$ & $1(12.5 \%)$ & 1 & $3(20.0 \%)$ & $4(13.3 \%)$ & 0.67 \\
\hline \multicolumn{7}{|l|}{ Pathophysiology } \\
\hline Initial surgery (dissection: degenerative) & $15: 0$ & $8: 0$ & 1 & $10: 5$ & $12: 18$ & 0.12 \\
\hline $\begin{array}{l}\text { Repeat surgery (pseudoaneurysm: } \\
\text { residual aneurysm) }\end{array}$ & $3: 12$ & $2: 6$ & 1 & $4: 11$ & $12: 18$ & 0.51 \\
\hline Previous surgery & & & 0.23 & & & 0.002 \\
\hline Ascending aorta replacement & $7(46.7 \%)$ & $1(12.5 \%)$ & & $4(26.7 \%)$ & $1(3.3 \%)$ & \\
\hline Aortic arch replacement & $2(13.3 \%)$ & $3(37.5 \%)$ & & $2(13.3 \%)$ & $17(56.7 \%)$ & \\
\hline Descending aorta replacement & $2(13.3 \%)$ & $3(37.5 \%)$ & & $5(33.3 \%)$ & $1(3.3 \%)$ & \\
\hline Thoracoabdominal aorta replacement & - & - & & $3(20.0 \%)$ & $5(16.7 \%)$ & \\
\hline $\begin{array}{l}\text { Aortic arch and thoracoabdominal aorta } \\
\text { replacement }\end{array}$ & - & - & & $1(6.7 \%)$ & $5(16.7 \%)$ & \\
\hline Partial arch replacement & $4(26.7 \%)$ & $1(12.5 \%)$ & & $0(0.0 \%)$ & $1(3.3 \%)$ & \\
\hline Surgical time (mins) & $510 \pm 114.1$ & $217 \pm 84.7$ & $<0.001$ & $476 \pm 155.5$ & $229 \pm 111.4$ & $<0.001$ \\
\hline \multicolumn{7}{|l|}{ Early outcomes } \\
\hline Acute kidney injury (\%) & $5(33.3 \%)$ & $0(0.0 \%)$ & 0.12 & $6(40.0 \%)$ & $1(3.3 \%)$ & 0.002 \\
\hline Spinal cord injury (\%) & $0(0.0 \%)$ & $1(12.5 \%)$ & 0.35 & $0(0.0 \%)$ & $1(3.3 \%)$ & 1 \\
\hline Infection (\%) & $5(33.3 \%)$ & $1(12.5 \%)$ & 0.37 & $5(33.3 \%)$ & $4(13.3 \%)$ & 0.14 \\
\hline Prolonged ventilation (\%) & $4(26.7 \%)$ & $0(0.0 \%)$ & 0.26 & $5(33.3 \%)$ & $2(6.7 \%)$ & 0.032 \\
\hline Cerebral infarction (\%) & $1(6.7 \%)$ & $0(0.0 \%)$ & 1 & $2(13.3 \%)$ & $0(0.0 \%)$ & 0.11 \\
\hline Vocal cord paralysis (\%) & $4(26.7 \%)$ & $0(0.0 \%)$ & 0.26 & $3(20.0 \%)$ & $0(0.0 \%)$ & 0.032 \\
\hline Sepsis (\%) & $1(6.7 \%)$ & $1(12.5 \%)$ & 1 & $1(6.7 \%)$ & $1(3.3 \%)$ & 1 \\
\hline ICU stay (days) & $3(2-4.5)$ & $1(1-2.3)$ & 0.006 & $4(2-8.5)$ & $1(1-2.0)$ & $<0.001$ \\
\hline Hospital stay (days) & $23(13-38)$ & $12.5(8.3-17.5)$ & 0.067 & $20(17-40.5)$ & $10(9-16.0)$ & $<0.001$ \\
\hline In hospital death (\%) & $0(0.0 \%)$ & $1(12.5 \%)$ & 0.35 & $0(0.0 \%)$ & $1(3.3 \%)$ & 1 \\
\hline
\end{tabular}

ously received ascending aorta replacement, repeat open surgery was performed by a redo median sternotomy. For other patients, repeat open repair of the aortic arch was performed by a left thoracotomy.

\section{Results}

The devices used for TEVAR were Najuta (Kawasumi Laboratories, Inc., Tokyo, Japan) $(\mathrm{n}=6)$, cTAG (W. L. Gore \& Associates, Inc., Flagstaff, AZ, USA) $(n=7)$, Relay (Bolton Medical, Inc., Sunrise, FL, USA) $(n=6)$,
TX2 (Cook Medical, Bloomington, IN, USA) $(n=10)$, Valiant (Medtronic, Minneapolis, MN, USA) $(\mathrm{n}=6)$, and Talent (Medtronic, Minneapolis, MN, USA) $(n=3)$. For repeat open surgery, nine patients underwent aortic arch replacement and six patients underwent descending aorta replacement for patients with dissected distal aorta. For patients without dissected distal aorta, 14 patients underwent aortic arch replacement and one patient underwent descending aorta replacement.

The demographics of the patients are shown in Table 1. For patients with dissected distal aorta, the time be- 


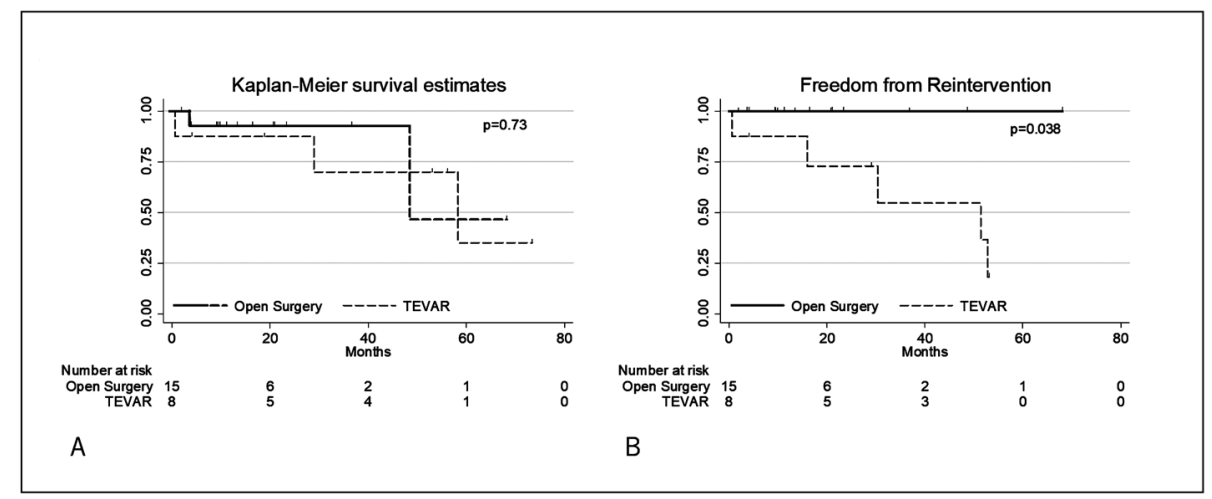

Fig. 3 Long-term outcomes and freedom from reintervention in patients with dissected distal aorta (entry closure group).

The dotted lines following each full (bold) line represent data outside standard error of $10 \%$.

tween repeat surgery and previous surgery was 36.5 months (q25-q75: 18.9-74.0). Of the five patients who underwent aortic arch replacement previously, one patient received frozen elephant trunk and one patient received conventional elephant trunk. There were no significant differences in age (open surgical repair [OSR]: $62 \pm 9.5$ years vs. TEVAR: $64 \pm 8.9$ years, $\mathrm{p}=0.66)$, and medications including angiotensin receptor blocker (OSR: 66.7\% vs. TEVAR: $62.5 \%, \mathrm{p}=1$ ), calcium channel blocker (OSR: $73.3 \%$ vs. TEVAR: $62.5 \%, \mathrm{p}=0.66)$, and beta blocker (OSR: $93.3 \%$ vs. TEVAR: $75.0 \%, p=0.27$ ). There also were no significant differences in other medical histories including hyperlipidemia (OSR: $26.7 \%$ vs. TEVAR: $50.0 \%, \mathrm{p}=0.37$ ), diabetes (OSR: $20.0 \%$ vs. TEVAR: $0.0 \%, \mathrm{p}=0.53$ ), and hypertension (OSR: $100.0 \%$ vs. TEVAR: $87.5 \%, \mathrm{p}=0.35$ ). However, surgical time was significantly longer in patients undergoing open surgical repair (OSR: $510 \pm 114.1 \mathrm{~min}$ vs. TEVAR: $217 \pm 84.7 \mathrm{~min}$, $\mathrm{p}<0.001)$. There was one patient with type $1 \mathrm{~b}$ endoleak, but there was no type 1a endoleak from previously implanted prosthetic graft.

For the early outcomes, open surgical repair was associated with longer ICU stay (OSR: 3, q25-q75 2-5 days, vs. TEVAR: $1, \mathrm{q} 25-\mathrm{q} 75$ 1-2 days, $\mathrm{p}=0.006)$. Although there was no statistical significance, hospital stay was also longer in open surgical repair (OSR: 23, q25-q75 13-38 days, vs. TEVAR: 13 , q25-q75 8-18 days, $\mathrm{p}=0.067$ ). There was only one in-hospital death from both groups and the cause of death was graft infection (Table 1).

For the long-term outcomes, there was no significant difference in long-term mortality $(p=0.73)$. However, TEVAR was associated with higher risk for reintervention $(p=0.038)$ (Fig. 3). Causes of deaths included graft infection $(\mathrm{n}=1)$, cerebral hemorrhage $(\mathrm{n}=1)$, pneumonia $(\mathrm{n}=1)$, leukemia $(\mathrm{n}=1)$, and included unknown cause $(n=1)$. For reintervention, four patients underwent endovascular repair due to residual endoleak $(n=1)$, stent graft induced new entry $(n=1)$, pseudoaneurysm $(n=1)$, and residual re-entry $(n=1)$. One patient underwent open surgical repair due to residual endoleak.

The demographics of the patients with non-dissected distal aorta are shown in Table 1 . The time between repeat surgery and previous surgery was 28.6 months (q25-q75 6.8-72.0). Of the 19 patients who underwent aortic arch replacement previously, two patients received conventional elephant trunk, but no patient received frozen elephant trunk. There were no significant differences in age (OSR: $68 \pm 10.4$ years vs. TEVAR: $70 \pm 7.9$ years, $p=0.52$ ), and medications including angiotensin receptor blocker (OSR: $46.7 \%$ vs. TEVAR: $50.0 \%, \mathrm{p}=1$ ), calcium channel blocker (OSR: $73.3 \%$ vs. TEVAR: $56.7 \%, \mathrm{p}=0.34$ ), and beta blocker (OSR: $60.0 \%$ vs. TEVAR: $60.0 \%$, $\mathrm{p}=1)$. There also were no significant differences in other medical histories including hyperlipidemia (OSR: 46.7\% vs. TEVAR: $53.5 \%, \mathrm{p}=0.76$ ), diabetes (OSR: $20.0 \%$ vs. TEVAR: $16.7 \%, \mathrm{p}=1$ ), and hypertension (OSR: $100.0 \%$ vs. TEVAR: $83.3 \%, p=0.15)$. However, surgical time was significantly longer in patients undergoing open surgical repair (OSR: $476 \pm 155.5 \mathrm{~min}$ vs. TEVAR: $229 \pm 111.4 \mathrm{~min}, \mathrm{p}<0.001)$. There was one patient with type $1 \mathrm{~b}$ endoleak, but there was no type $1 \mathrm{a}$ endoleak from previous implanted prosthetic graft.

For the early outcomes, incidence of AKI (OSR: $40.0 \%$ vs. TEVAR: $3.3 \%, \mathrm{p}=0.002)$, prolonged ventilation (OSR: $33.3 \%$ vs. TEVAR: $6.7 \%, p=0.032$ ), and vocal cord paralysis (OSR: $20.0 \%$ vs. TEVAR: $0.0 \%, \mathrm{p}=0.032$ ) were more often observed in open surgical repair than in patients treated by TEVAR. Furthermore, open surgical repair was associated with longer ICU stay (OSR: 4 q25-q75 2-9 days, vs. TEVAR: 1, q25-q75 1-2 days, $\mathrm{p}<0.001$ ) and hospital stay (OSR: 20, q25-q75 17-41 days, vs. TEVAR: 10, q25-q75 9-16 days, $\mathrm{p}<0.001$ ). There was only one in-hospital mortality from both groups and the cause of death was graft infection. 


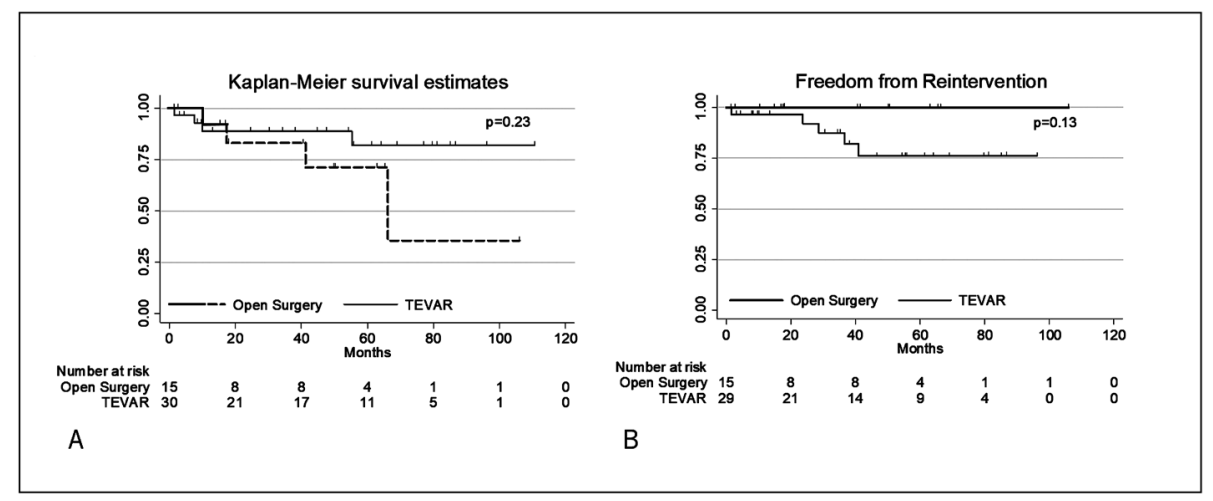

Fig. 4 Long-term outcomes and freedom from reintervention in patients with non-dissected distal aorta (aneurysm exclusion group).

The dotted lines following each full (bold) line represent data outside standard error of $10 \%$.

For the long-term outcome, there was no significant difference in long-term mortality $(p=0.23)$. Although there was no statistical significance, freedom from reintervention $(p=0.13)$ tended to be lower in the TEVAR group (Fig. 4). Causes of death included cerebral hemorrhage $(n=3)$, pneumonia or other infections $(n=3)$, and unknown cause $(n=2)$. For reintervention, five patients underwent endovascular reintervention due to type 2 endoleak $(n=2)$, type $1 \mathrm{~b}$ endoleak $(n=1)$, stent migration $(\mathrm{n}=1)$, and residual aneurysmal rupture $(\mathrm{n}=1)$. One patient underwent open surgical repair due to pseudoaneurysm.

\section{Discussion}

In patients undergoing repeat thoracic aortic repair from previous anastomosis site, patients who underwent open surgical repair were more likely to develop postoperative AKI, vocal cord paralysis, and experience prolonged ventilation. Using previously implanted prosthetic graft as a landing zone for TEVAR was not associated with risk for type 1 endoleak. ICU stay was significantly shorter in patients who underwent TEVAR. Although there were no significant differences in long-term survival, TEVAR in patients with dissected landing zone was associated with higher incidence of reintervention.

The intraoperative and in-hospital mortality of the patients who needed redo surgery for thoracic aortic aneurysm has been reported to be $7.5 \%$ and $17.3 \%$, respectively. ${ }^{2)}$ The multivariate analysis from 11 European aortic centers showed that older age at redo surgery was an independent risk factor for in-hospital mortality. ${ }^{2)}$ Redo surgery is associated with technical difficulties mostly due to dissection of adhesion from previous surgery. Endovascular technology provides a new approach in treating this set of patients, in which aortic repair could be performed without redo thoracotomy, thus avoiding a need for dis- secting adhesion and preventing injury of the surrounding tissues such as lung and esophagus.

Endoleak, a phenomenon specific to endovascular therapy, could be a major concern for repeat TEVAR as it may be associated with further reintervention. ${ }^{7}$ In our series, there was no type $1 \mathrm{a}$ endoleak after repeat TEVAR, which showed that using previously implanted prosthetic graft as a landing zone was acceptable. However, freedom from reintervention was significantly lower in patients with dissected landing zone undergoing TEVAR, which may be similar to a pathophysiology of a chronic type B dissection.

In the meta-analysis of treatment of chronic type B aortic dissection, ${ }^{8)}$ in hospital mortality rate was better in TEVAR compared to in hospital mortality rate for open surgical repair (3.2\% vs. $9.6 \%)$. Postoperative incidence of paraplegia $(0.43 \%$ vs. $4.8 \%)$ and stroke $(0.82 \%$ vs. $5.8 \%$ ) was also less observed in TEVAR compared to open surgical repair. However, in the propensity adjusted analysis, three years freedom from reintervention was $96.7 \%$ in open surgical repair compared to $87.5 \%$ in TEVAR. ${ }^{9)}$ Similarly, early reintervention rate of $15 \%$ to $22 \%$ has been reported in patients undergoing TEVAR for chronic type B dissection. ${ }^{10-12)}$

Sealing entry site by TEVAR is expected to induce aortic remodeling, while persistent false lumen perfusion is reported to be an independent risk factor for progressive aortic enlargement and adverse long-term outcomes. ${ }^{13)}$ Endoluminal exclusion of acute type B dissection without endoleak is associated with $80 \%$ volume increase of the true lumen and $86 \%$ reduction in false lumen at five years after surgery. ${ }^{14)}$ However, complete false lumen thrombosis in chronic aortic dissection occurs only in $40 \%$ to $80 \%$ of the patients at the level of the device and in $0 \%-40 \%$ of the patients at the level distal to the device. ${ }^{15)}$ In a prospective cohort study, VIRTUE registry revealed that patients with subacute dissection (15-92 days) demon- 
strated a similar degree of aortic remodeling compared to patients with acute dissection ( $<15$ days). ${ }^{16)}$ However, the rate of remodeling was poorer in the chronic phase $(>92$ days). ${ }^{16)}$ In our series, TEVAR in patients with dissected distal aorta was performed at 49.3 months (q25-q75 IQR 9.5-82.9) after the previous surgery. Thus, remodeling of the distal aorta may have been unfavorable. In the metaanalysis of 15 studies, Gambardella et al. reported that type B dissection was the most common pathology for secondary open procedure following TEVAR. ${ }^{7}$ Decreased long-term survival has also been reported in patients without remodeling after TEVAR in chronic type B aortic dissection. ${ }^{17)}$ Although our study has shown similar longterm survival in patients undergoing TEVAR and open surgery, this might have been achieved due to close followup on these patients proceeded when necessary by prompt reintervention procedures.

There are several limitations to this study. This is a retrospective study with a small sample size. Prospective trials with larger samples size should be performed to confirm the results. Furthermore, repeat TEVAR in this study was performed at a chronic phase of aortic dissection. Repeat TEVAR performed at a more acute phase may show better outcomes.

\section{Conclusion}

Using previously implanted prosthetic graft was acceptable as a landing zone for repeat TEVAR. Although early outcomes were better in TEVAR, incidence of reintervention was higher in patients with dissected distal aorta undergoing TEVAR. Although similar long-term survival may be achieved via a close follow-up, repeat TEVAR in patients with chronic dissected landing zone should be considered due to the high incidence of reintervention.

\section{Acknowledgments}

This research was supported by Jichi Medical University Young Investigator Award (DH).

\section{Disclosure Statement}

Atsushi Yamaguchi serves as a consultant to Japan Lifeline Inc.; Daijiro Hori, Koichi Yuri, Sho Kusadokoro, Hiroyasu Katayama, and Naoyuki Kimura have no conflict of interest.

\section{Author Contributions}

Study conception: DH, KY

Data collection: DH, SK, HK

Analysis: DH, NK
Investigation: $\mathrm{DH}$

Writing: DH

Funding acquisition: $\mathrm{DH}$

Critical review and revision: all authors

Final approval of the article: all authors

Accountability for all aspects of the work: all authors

\section{References}

1) Moz M, Misfeld M, Leontyev S, et al. Aortic arch reoperation in a single centre: early and late results in 57 consecutive patients. Eur J Cardiothorac Surg 2013; 44: e82-6.

2) Luehr M, Peterss S, Zierer A, et al. Aortic events and reoperations after elective arch surgery: incidence, surgical strategies and outcomes. Eur J Cardiothorac Surg 2018; 53 : 519-24.

3) Kamman AV, Jonker FHW, Nauta FJH, et al. A review of follow-up outcomes after elective endovascular repair of degenerative thoracic aortic aneurysms. Vascular 2016; 24 : 208-16.

4) Canaud L, Gandet T, Ozdemir BA, et al. Hybrid aortic repair of dissecting aortic arch aneurysm after surgical treatment of acute type A dissection. Ann Vasc Surg 2016; 30: 175-80.

5) Moulakakis KG, Mylonas SN, Markatis F, et al. A systematic review and meta-analysis of hybrid aortic arch replacement. Ann Cardiothorac Surg 2013; 2: 247-60.

6) Roy AK, Mc Gorrian C, Treacy C, et al. A comparison of traditional and novel definitions (RIFLE, AKIN, and KDIGO) of acute kidney injury for the prediction of outcomes in acute decompensated heart failure. Cardiorenal Med 2013; 3: 26-37.

7) Gambardella I, Antoniou GA, Torella F, et al. Secondary open aortic procedure following thoracic endovascular aortic repair: meta-analytic state of the art. J Am Heart Assoc 2017; 6: e006618.

8) Thrumurthy SG, Karthikesalingam A, Patterson BO, et al. A systematic review of mid-term outcomes of thoracic endovascular repair (TEVAR) of chronic type B aortic dissection. Eur J Vasc Endovasc Surg 2011; 42: 632-47.

9) van Bogerijen GHW, Patel HJ, Williams DM, et al. Propensity adjusted analysis of open and endovascular thoracic aortic repair for chronic type B dissection: a twenty-year evaluation. Ann Thorac Surg 2015; 99: 1260-6.

10) Resch TA, Delle M, Falkenberg M, et al. Remodeling of the thoracic aorta after stent grafting of type B dissection: a Swedish multicenter study. J Cardiovasc Surg (Torino) 2006; 47: 503-8.

11) Parsa CJ, Williams JB, Bhattacharya SD, et al. Midterm results with thoracic endovascular aortic repair for chronic type B aortic dissection with associated aneurysm. J Thorac Cardiovasc Surg 2011; 141: 322-7.

12) Andacheh ID, Donayre C, Othman F, et al. Patient outcomes and thoracic aortic volume and morphologic changes following thoracic endovascular aortic repair in patients with complicated chronic type B aortic dissection. J Vasc Surg 2012; 56: 644-50; discussion, 650.

13) Tsai TT, Evangelista A, Nienaber CA, et al. Partial thrombosis of the false lumen in patients with acute type B aortic dissection. N Engl J Med 2007; 357: 349-59. 
14) Kim KM, Donayre CE, Reynolds TS, et al. Aortic remodeling, volumetric analysis, and clinical outcomes of endoluminal exclusion of acute complicated type B thoracic aortic dissections. J Vasc Surg 2011; 54: 316-24; discussion, 324-5.

15) Conway AM, Qato K, Mondry LR, et al. Outcomes of thoracic endovascular aortic repair for chronic aortic dissections. J Vasc Surg 2018; 67: 1345-52.
16) Mid-term outcomes and aortic remodelling after thoracic endovascular repair for acute, subacute, and chronic aortic dissection: the VIRTUE Registry. Eur J Vasc Endovasc Surg 2014; 48: 363-71.

17) Mani K, Clough RE, Lyons OT, et al. Predictors of outcome after endovascular repair for chronic type B dissection. Eur J Vasc Endovasc Surg 2012; 43: 386-91. 